\title{
Is rectal administration an alternative route for imatinib?
}

\author{
Nielka P. H. van Erp • Roos L. Oostendorp • \\ Henk-Jan Guchelaar • Jan H. M. Schellens • \\ Hans Gelderblom
}

Received: 2 October 2006 / Accepted: 18 December 2006 / Published online: 8 February 2007

(C) Springer-Verlag 2007

\begin{abstract}
A 52-year-old woman with metastatic gastro-intestinal stromaceltumor (GIST) presented herself in March 2006 with tumor-related intra-abdominal obstructions and diffuse intra-abdominal bleeding. Priorly, the metastatic GIST was successfully treated with $400 \mathrm{mg}$ imatinib since 2002 but now appeared to be progressive again. The patient underwent palliative resection of multiple bleeding peritoneal tumor deposits. When confronted with GIST progression; as seen in this patient, the dose of imatinib should be elevated from 400 to $800 \mathrm{mg} /$ day [1]. However, a major limitation for treatment in this patient was that, due to the gastrointestinal obstructions, she was unable to take anything orally, including the imatinib tablets for 8 days prior to surgery. Unfortunately, imatinib is available as a tablet formulation only. Therefore, in this patient we tested the rectal route of administration as an alternative way to administer the drug.
\end{abstract}

N. P. H. van Erp · H.-J. Guchelaar

Department of Clinical Pharmacy and Toxicology,

Leiden University Medical Center,

Leiden, The Netherlands

R. L. Oostendorp · J. H. M. Schellens

Division of Experimental Therapy and Medical Oncology, The Netherlands Cancer Institute/Antoni van Leeuwenhoek Hospital, Amsterdam, The Netherlands

N. P. H. van Erp ( $\square)$

Albinusdreef 2, Postbox 9600, 2300 RC,

Postal zone L0-P Leiden, The Netherlands

e-mail: p.h.van_erp@lumc.nl

H. Gelderblom

Department of Clinical Oncology,

Leiden University Medical Center,

Leiden, The Netherlands
The day following surgery, the patient received imatinib $400 \mathrm{mg}$ b.i.d. with the imatinib oral tablets being administered rectally. After the fourth dose of imatinib given rectally we collected blood samples at $t=0,1,2,3,4,5,6,8$ and $10 \mathrm{~h}$. The patient volunteered in a pharmacokinetic study a year before [2]. In the study, after informed consent, we collected steady state blood levels of imatinib at the same time points as described above, but after an oral dose of $400 \mathrm{mg}$ imatinib. This enabled us to compare the area under the concentration-time (AUC) curve following oral and rectal administration of imatinib in this patient. Plasma concentrations of imatinib were analyzed at The Netherlands Cancer Institute by a validated HPLC-UV assay with a variation coefficient within the generally accepted $15 \%$ range and a lower limit of quantification of $10 \mathrm{ng} / \mathrm{ml} . \mathrm{AUC}_{0-10 \mathrm{~h}}$ after the oral administration of $400 \mathrm{mg}$ imatinib was 35,508 and it was $14,243 \mathrm{ng} / \mathrm{ml} \mathrm{h}$ after rectal administration (Fig. 1) calculated by the trapezoidal method. Assuming relatively small intraindividual variation in pharmacokinetics, comparison of the AUCs indicates that at least $40 \%$ of the oral imatinib levels are reached by rectal administration. About $40 \%$ will be a slight underestimation because steady-state conditions were not fully reached. The AUC after the fourth rectal dose was estimated at $80-90 \%$ of the steady-state AUC.

The $t_{1 / 2}$ of imatinib is $\approx 18 \mathrm{~h} \mathrm{[3].} \mathrm{In} \mathrm{the} 9$ days before rectal administration of imatinib the body is cleared from imatinib. Therefore, the AUC measured after the fourth rectal dose of imatinib is solely produced by absorbance of imatinib from the rectum and is not influenced by the oral dose used before.

The lack of alternative dosing forms of imatinib sometimes causes problems in clinical practice. 


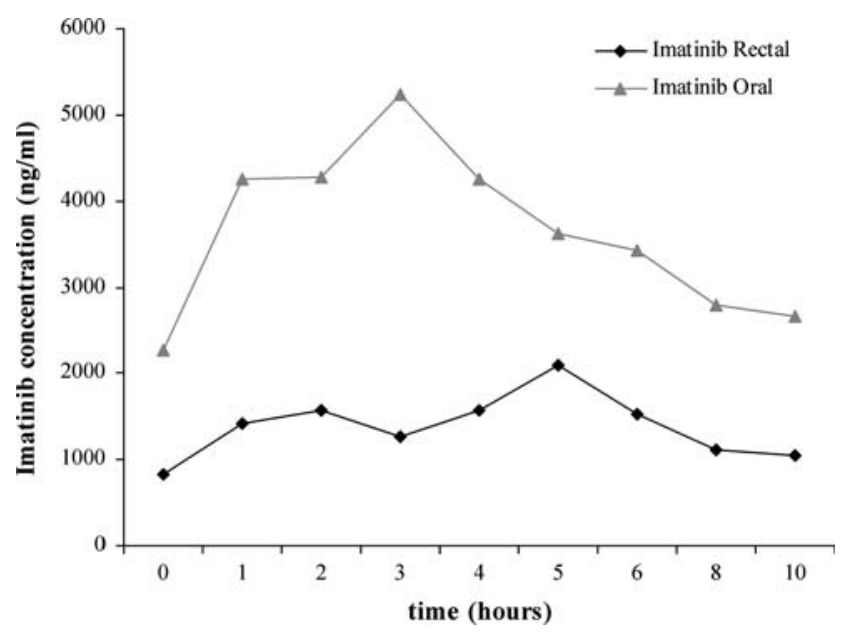

Fig. 1 Representative plasma concentrations versus time profile of imatinib after rectal or oral administration of $400 \mathrm{mg}$

Patients with GIST may show obstruction or narrowing of the gastro-intestinal tract causing problems to take food and drugs orally. These patients are unable to take imatinib treatment. Based on our observation, in these circumstances, rectal administration of a double dose of imatinib could be a good alternative. Imatinib mesylate is a highly water soluble drug with a bioavailability of nearly $100 \%$ when taken orally [4]. This characteristic readily predicts absorption from the rectal mucosa. Indeed, in the patient presented here, we demonstrated by plasma level measurement that imatinib could be administered rectally resulting in a $40 \%$ drug exposure. Therefore, doubling the dose is anticipated to reach a similar drug exposure compared to when given orally.

\section{References}

1. Verweij J, Casali PG, Zalcberg J, LeCesne A, Reichardt P, Blay JY et al (2004) Progression-free survival in gastrointestinal stromal tumours with high-dose imatinib: randomised trial. Lancet 364(9440):1127-1134

2. van Erp NP, Gelderblom H, Li JZ, Zhao M, Ouwerkerk J, Nortier JW et al (2006) Influence of cytochrome P-450 3A4 inhibition on the steady-state pharmacokinetics of imatinib (submitted)

3. Judson I, Ma P, Peng B, Verweij J, Racine A, di Paola ED et al (2005) Imatinib pharmacokinetics in patients with gastrointestinal stromal tumour: a retrospective population pharmacokinetic study over time. EORTC Soft Tissue and Bone Sarcoma Group. Cancer Chemother Pharmacol 55(4):379-386

4. Peng B, Dutreix C, Mehring G, Hayes MJ, Ben Am M, Seiberling M et al (2004) Absolute bioavailability of imatinib (Glivec) orally versus intravenous infusion. J Clin Pharmacol 44(2):158-162 